\title{
A wearable embedded device for chronic low back patients to track lumbar spine position.
}

\author{
Maheswaran Shanmugam*, Sunilvignesh Nehru, Sathesh Shanmugam \\ Department of Electronics and Communication Engineering, Kongu Engineering College, Tamil Nadu, India
}

\begin{abstract}
In present scenario, people always work in an enclosed environment where they sit in the bend posture that causes severe damage in lumbar spine. According to the survey of World Health Organization, around the world 500,000 to 800,000 people are suffering from spinal cord injury (SCI). In that about $53 \%$ of the people who are aged between 16 and 30 suffer from chronic low back pain. A new wearable device which consists of miniature gyroscope and accelerometer are used in detecting the voluntary action of lumbar spine in the proposed system. The proposed system consists of a vibrator, Bluetooth element, a microcontroller and an android application. The vibrator motor is used to intimate the improper sitting position of the person. A Bluetooth element is used in transmitting the data from the module and the android application that runs on smart phone. It records the analyzed data of the sensor. A microcontroller is used to control the overall working of the designed system. The capability of this cost effective, simple device is capable to measure the lumbar spine position with the efficiency of 95\% and provide a feedback to the user.
\end{abstract}

Keywords: Spinal cord injury, Chronic low back pain, Accelerometer, Bluetooth module, Android application. Accepted on June 13, 2017

\section{Introduction}

The healthcare industry is facing a growing emphasis on healthcare management instead of conventional disease management [1]. SITTING is one among the most common postures [2-7] of human beings in life and reported that people sit for six hours each day. Current healthcare information modules are designed for monitoring the posture for low back pain patients. Chronic Low Back Pain (CLBP) is a prominent cause of disability dominant in similar parts across various countries and cultures [2]. One in five adults suffers from CLBP annually. In industrialized cultures, chronic pain is fast becoming the greatest health problem which embraces low back pain costs of US\$100-\$200 billion annually [4]. About $75 \%$ to $85 \%$ of workers nonappearance is credited to recurrent and chronic back pain [6]. Presently some of the methods like e-cushion and SQUID Bio magnetometer system are proposed as the solution of spinal cord injury. In real time, wireless networks are used to transmit the medical data with secured protection on cloud service. In this process, the private medical data cannot be disclosed by an individual in the middle attack. Thus, the collected data would not be threatened and can be made use of consistent refuge plan by healthcare service provider (HSP). The Advanced Encryption Standard (AES) is used to refuge the framework on system [5]. The healthcare applications could support the privacy easily [8-20]. The framework advanced for a module embattled by low power microcontroller within real time embedded operating system. The classification of spinal cord is shown in the Figure 1.

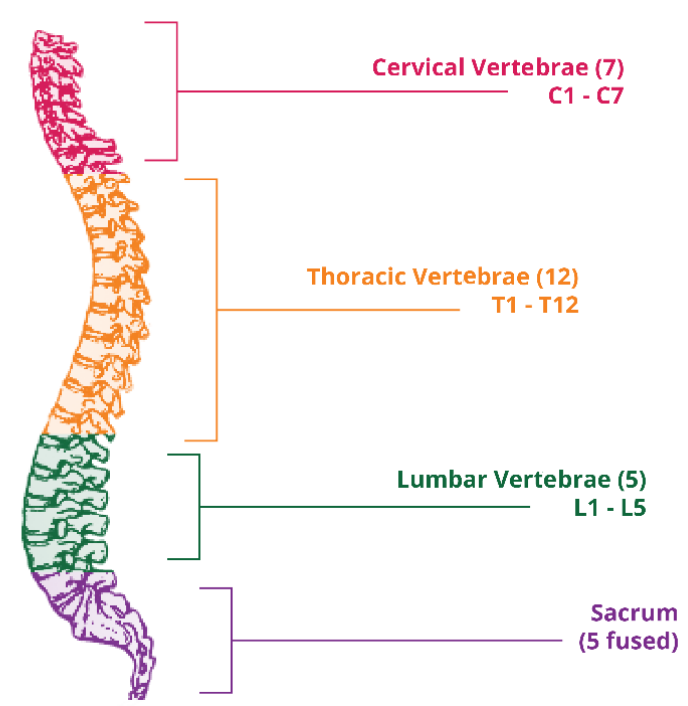

Figure 1. Classification of spinal cord.

The following are the related work on the field of healthcare lumbar spine posture analysis. Chhikara et al. [1] observed that IMPAIRED (In-House Monitoring Low back Pain Related Disability) concept will enable accurate assessment of chronic LBP patients, monitors the treatment effects on patients, checks on whether patients follow treatment plans and objective assessment of the clinical effectiveness of current and original treatments. The key advantage is that the product will have an integrated approach towards disability assessment and 
applicability to multiple patient groups through relatively minor modifications.

Ladenbauer et al. [11] developed an innovative approach to have a root fiber with implanted electrodes to excite lumbar posterior. The cathode position determines the entry of the posterior root fibers at the spinal cord portions which are categorized by 1) A strong curvature of the fiber, and 2) A border between anatomical structures with significantly different conductivities. Efficient excitation of the different structures with simple noninvasive method on rehabilitation with motor disorders helps in identifying the position of the spinal cord. $\mathrm{Hu}$ et al. [21] presented the benefits of both low cost sensor and wireless sensors. The circuits were incorporated into the sole of the cushion. The designed system subjected on power and reliability and could be improved through modifications of the data sampling rate, communication frequency and the analysis algorithms running on the handset. The smart cushion for back prevention based on similar frame works was also being used.

Bai et al. [19] observed that the accelerometer sensor could be used to design and implement a fall monitor with mobility detection. The design was based on the smart phone which could be carried anywhere including out of doors. The GPS in the smart phone was used to detect where the fall had taken place. The acceleration values of $\mathrm{X}, \mathrm{Y}$ and $\mathrm{Z}$ axis were used for the static state analysis of the falling position. The experimental results showed an overall accuracy of smart gadget over various sitting positions to be $75.9 \%$. Xu et al. [18] developed a textile-based sensor used to analyze the sitting posture of human. The experimental results showed upto $89 \%$ of accuracy and concluded that the monitoring of postures were more economical and comfort for the people.

Samiei-Zonouz et al. [15] observed that the growing powerful embedded sensors such as accelerometers, gyroscopes, microphones, and cameras were useful for monitoring of various parameters in different applications. The design and implementation of systems for monitoring the human posture using a smartphone-centric software with acceleration sensor which was suitable for healthcare applications. The position and the activity monitoring of human parts had its application in the field of medical advice, context awareness, calorieburning guides, etc. The obtained results were demonstrated and presented instead of using distributed wearable sensors over the human body; a smartphone incorporating its embedded sensors (i.e., acceleration sensors) was utilized for performing posture and activity monitoring. The proposed module uses non-radiative sensors to detect the voluntary movement of lumbar spine which transfers the data to the user through wireless communication. The vibrator motor intimates the improper sitting position of the person. The android application records the analyzed data from the sensor and alerts the people about their position.

\section{Methodology and System Design}

The most common effects found in Chronic Low Back Pain patients are a change in the movement of the back, difficulty in having a restful sleep and experience of fatigueless. These effects can be severe and can alter the ability of CLBP patients to perform simple day to day activities and lead an independent life.

Therefore the wearable prototype has be developed to monitor the movement of the lumbar spine in the activity pattern. The proposed system design shown in Figure 2 consists of two units: position analysis and wireless communication. The position analysis unit has an accelerometer sensor to frequently get the acceleration's raw values. The wireless communication unit has the Bluetooth module that transmits the data to the mobile phone.

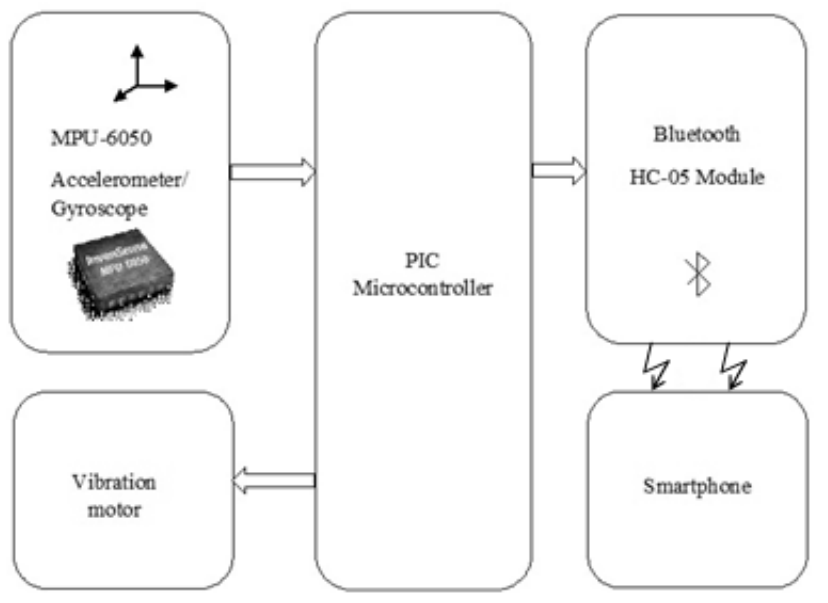

Figure 2. System design.

\section{Position analysis unit}

An accelerometer measures the acceleration values. Accelerometer is used in various applications like industry and science. The MPU-6050 device is a silicon chip which is a combination of 3-axis gyroscope and a 3-axis accelerometer [4], with an on-board Digital Motion Processor (DMP), which includes 6-axis Motion Fusion algorithms [10].

Table 1. MPU-6050 specification.

\begin{tabular}{lcll}
\hline \multicolumn{2}{c}{ Accelerometer } & \multicolumn{2}{c}{ Gyroscope } \\
\hline $\begin{array}{l}\text { Scale range } \\
(\mathrm{g})\end{array}$ & $\begin{array}{l}\text { Sensitivity } \\
(\mathrm{LSB} / \mathrm{g})\end{array}$ & $\begin{array}{l}\text { Scale range } \\
\left({ }^{*} / \mathrm{sec}\right)\end{array}$ & $\begin{array}{l}\text { Sensitivity } \\
\left(\mathrm{LSB} /{ }^{*} / \mathrm{sec}\right)\end{array}$ \\
\hline \pm 2 & 16384 & \pm 250 & 131 \\
\hline \pm 4 & 8192 & \pm 500 & 65.5 \\
\hline \pm 8 & 4096 & \pm 1000 & 32.8 \\
\hline \pm 16 & 2048 & \pm 2000 & 16.4 \\
\hline
\end{tabular}

The module is accessed through an assisting master $\mathrm{I}^{2} \mathrm{C}$ bus, and allows the system to collect a full set of sensor data. The InvenSense Platform has the MPU-6050 that extracts motion- 
based complexities and discharges sensor management from the operating system. Table 1 shows the sensitivity range for the corresponding scale range (i.e. if we use \pm 2 scale range the sensitivity value is 16384).

\section{Wireless communication unit}

The system needs a wireless module for data transmission. HC-05 is a Bluetooth module for exchanging of data through wireless technology for a shorter distance. This Bluetooth version 2 transmits the data at the rate of $3 \mathrm{Mbps}$ through serial communication and follows the ISM band of $2.4 \mathrm{GHz}$ transceiver. This baseband module employs CSR Blue core 04 and employs CMOS technology with AFH (Adaptive Frequency Hopping). The low power module cc2541 uses SMD BLE V.4.0.

\section{Warning system}

The module consists of LED notifications and vibration alerting unit. LED notification is used during power on, pairing devices, on low battery and transmitting the data. Vibration unit alerts the user, when they are in a bad posture. The vibration unit has a tiny shaft-less vibratory motor which is a perfect non-audible indicator.

\section{Sensor placement}

The hardware module is shown in the Figure 3. 1. consists of 1.MPU-6050 sensor, 2. Bluetooth module HC-05 and 3. battery.

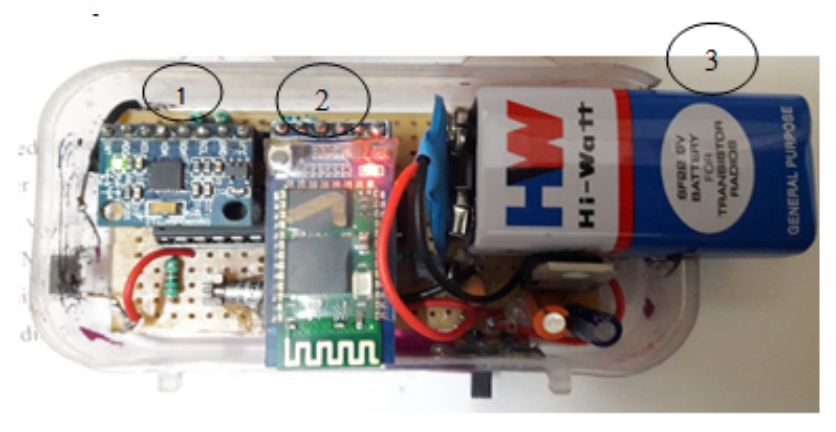

Figure 3. Hardware design.

The sensor module can be placed on the lower back or at the shirt pocket. Initially, the module is positioned near lumbar spine, the reference value is set with the help of a button according to the persons [18]. When the user undergoes bad posture, the alert notification is received through mobile phone. After making use of miniature sensors, the device has been minimized and is placed in the shirt pocket. Again the same analysis was calculated as for device placed in lumbar position. The Figure 4 shows the placement of the sensor module.

\section{Power ratings}

The designed hardware unit consumes $0.4 \mathrm{~W}$ of power. The modules are shown in Figure 5 with their ratings. The Figure 5 relates the different modules power consuming in watts. The different modules are MPU6050, microcontroller, Bluetooth module and vibration motor.

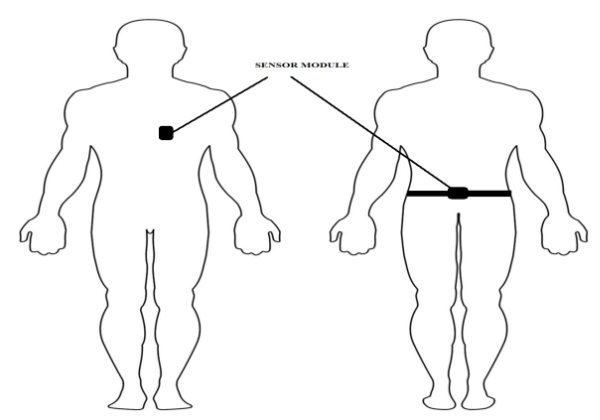

Figure 4. Sensor placement.

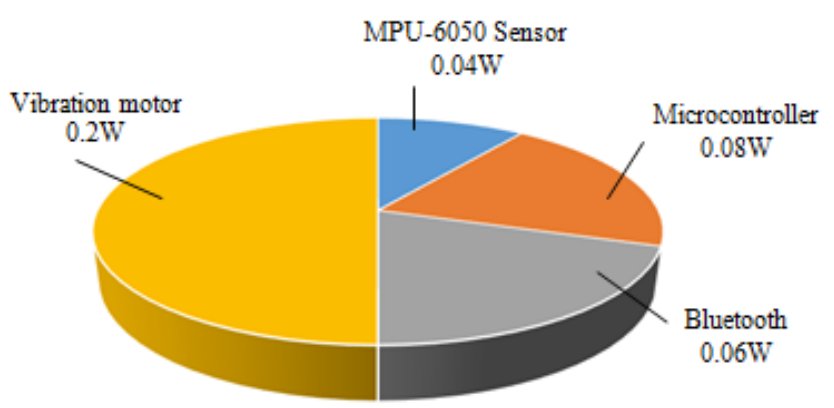

Figure 5. Power analysis.
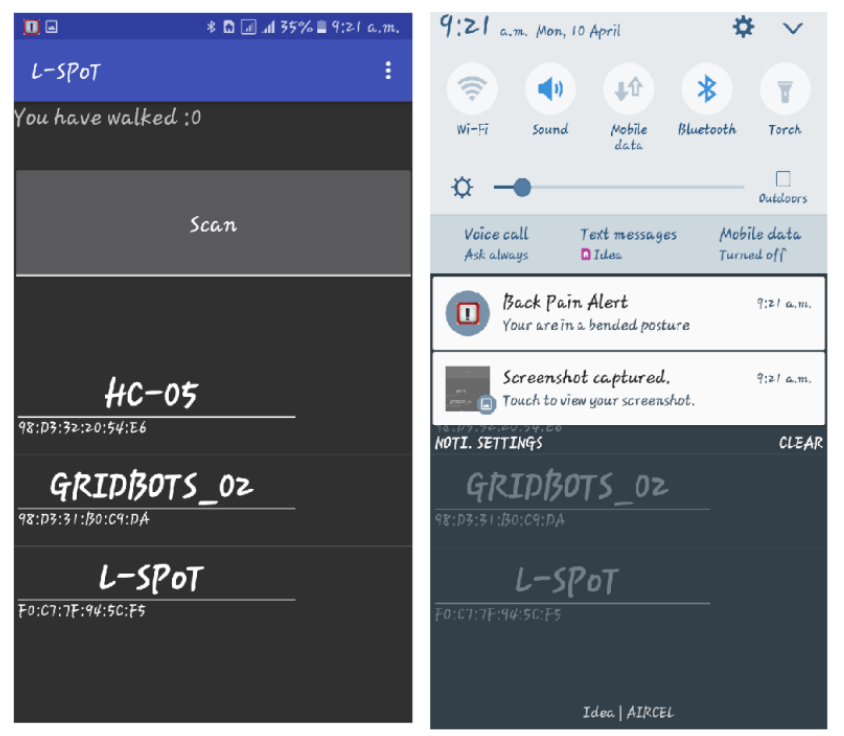

Figure 6. Android application.

\section{User interface}

Android application helps the user to have visual feedback via graphical user interface (GUI) and real time data processing unit. This application helps the user or doctor to specify the preferences for a number of parameters and thresholds. The doctor can use the graphical display to monitor the patient even if he is at distance using internet or SMS by visually and easily tracking the patient's status [22]. The graphical display is a form of an animation figure that mirrors the posture and 
position of the user in real time as shown in Figure 6. The application menu offers the facility to configure the sensors. The sensor settings such as sampling rate, sensibility $( \pm \mathrm{g})$, and sensor type (accelerometer, gyroscope) and the size of the processing window can be configured. It is also possible to enable and disable the logging process.

\section{Results and Discussion}

Experiment was conducted to find the position of the spinal cord using the set analysis and the data were received in the android application.

\section{Study}

The hardware module is designed and tested with different persons. According to their posture analysis the reference value and acceleration value are calculated. From the calculated value the set point/threshold value of the bend posture is studied.

Table 2. Reference and acceleration values.

\begin{tabular}{lllllll}
\hline Persons & \multicolumn{3}{l}{ Reference Value } & \multicolumn{3}{l}{ Acceleration Value } \\
\cline { 2 - 7 } & \multicolumn{2}{l}{ Straight Position } & \multicolumn{3}{l}{ Forward Bend } \\
\cline { 2 - 7 } & RX & RY & RZ & AX & AY & AZ \\
\hline Person 1 & 206 & 4352 & 389 & 321 & 4001 & 1435 \\
\hline Person 2 & 279 & 3949 & 140 & 305 & 3897 & 1354 \\
\hline Person 3 & 352 & 4196 & 575 & 543 & 3701 & 1811 \\
\hline Person 4 & 537 & 4110 & 234 & 494 & 3885 & 1380 \\
\hline Person 5 & 440 & 4104 & 533 & 334 & 3888 & 1547 \\
\hline Person 6 & 473 & 3992 & 632 & 491 & 3758 & 1809 \\
\hline Person 7 & 247 & 4107 & 275 & 342 & 3755 & 1866 \\
\hline Person 8 & 631 & 4024 & 370 & 730 & 3822 & 1601 \\
\hline Person 9 & 451 & 3987 & 489 & 563 & 4012 & 1532 \\
\hline Person 10 & 491 & 4004 & 589 & 501 & 4009 & 1645 \\
\hline
\end{tabular}

From Table 2, the different person acceleration values are calculated and analysis of the set value is shown in Figure 7. From the study of axis of hardware module placed near the front shirt pocket, the persons forward and backward positions are represented in the z-axis. With the help of these samples the forward bend posture range is analyzed and the acceleration limit value is set.

\section{Achieved result}

After the analysis of the set value, the limitation of the acceleration value is fixed. Different postures of a single person are taken with respect to time(s). Start with the reference straight position followed by acceleration movement value are taken respectively, if the difference between reference value and acceleration value is greater than 1000 , it seems to be a bend posture. Z-axis refers to the forward movement. In Figure 8 it is shown that at the time of 40 th second, the $\mathrm{z}$ axis value gets increased, it exceeds 1000 . The warning unit gets alert using the vibration motor. After $75^{\text {th }}$ second, it starts to decrease and the warning unit gets off.

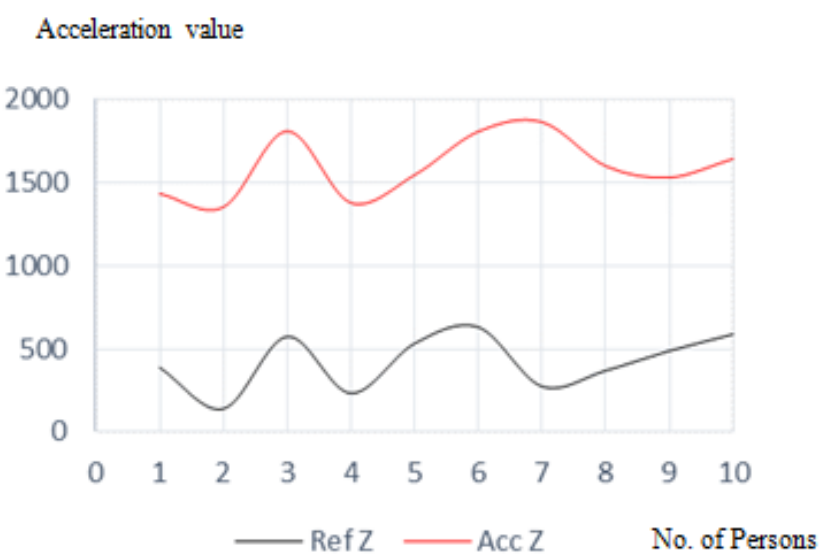

Figure 7. Forward bend analysis.

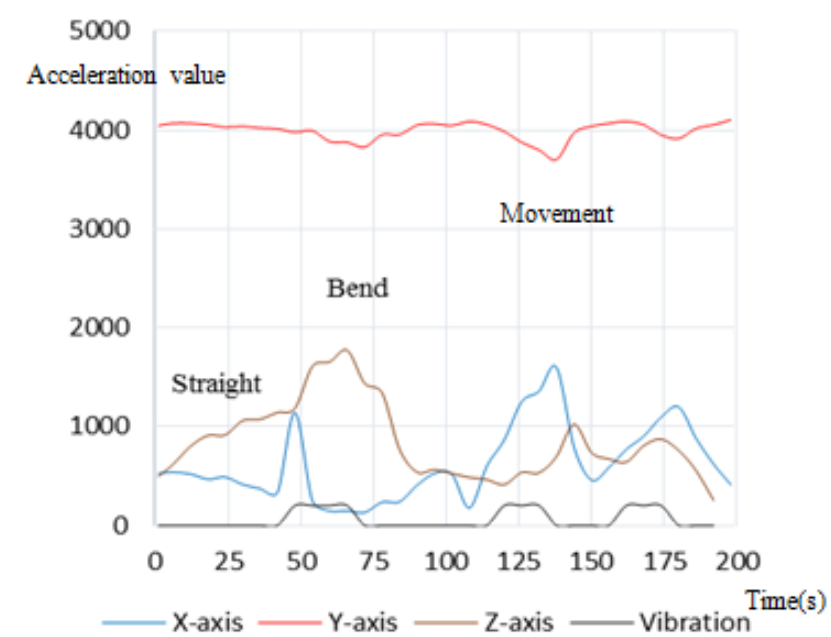

Figure 8. Person's position analysis.

Acceleration angle for the forward posture is measured with raw data and is converted into angle with the help of map function.

$\operatorname{Map}_{\mathrm{a}}=($ Acc, minval, maxval, inmin, inmax $) \rightarrow(1)$

Map $_{\mathrm{a}}$ refers to the all $\mathrm{x}, \mathrm{y}$ and $\mathrm{z}$ axis. Acc is related to the raw data from the sensor. Minval and maxval can be used to set the range of the acc values and inmin, inmax are used to fix the output range. Constrain values must be between ranges, though out-of-range values may be sometimes useful.

According to the sensor, the forward bend values are the changes with respect to right triangle and the angle measurement tangent inversion are used which is called as arctangent or atan2.

$A c c_{\mathrm{fwd}}=\left(\operatorname{atan}_{2}\left(-\operatorname{Map}_{\mathrm{y}},-M_{\mathrm{a}}\right)^{*} R D\right) \rightarrow(2)$

The equation 2 shows the forward movement, RD refers to the radian to degree conversion and is equal to 57.324840 . 

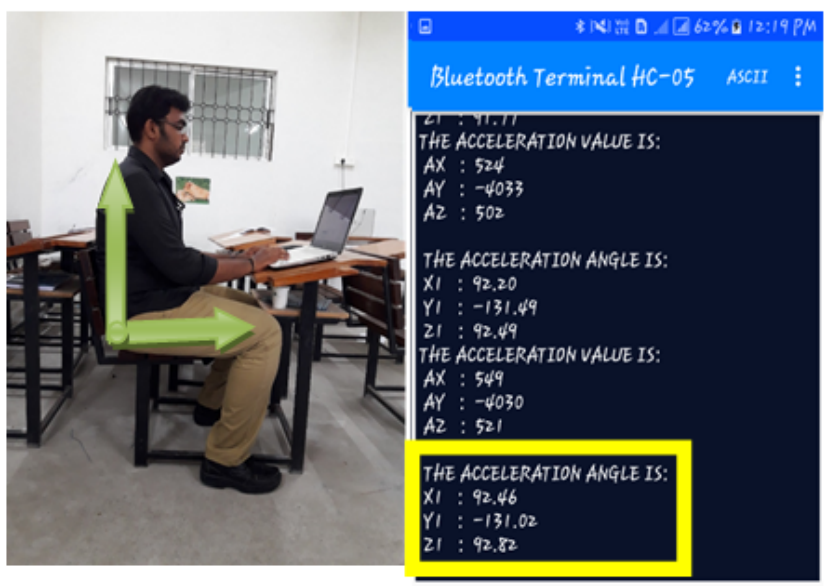

Figure 9. Straight position.

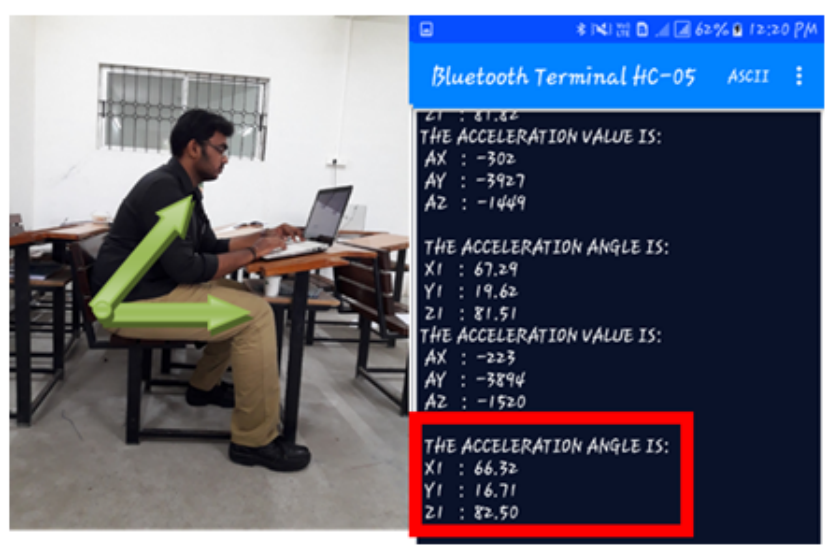

Figure 10. Bad posture.

The Figures 9 and 10 shows the perfect straight posture and bad posture of the person with respect to the angle. The good posture indicates the angle $90-110^{\circ}$. And bad posture indicates below $70^{\circ}$.

\section{Gravitational acceleration}

For a person, different gravitational forces are applied on standing, walking and sitting position. Gravity is measured using the acceleration with respect to the data and sensitivity selection range. Different spikes in the Figure 11 show the walking position.

Gravity $_{A C C}=\frac{\text { Accelerationon Value }}{\text { Sensitivity }} \times 9.81 \mathrm{~m} / \mathrm{s}^{2} \rightarrow(3)$

\section{Gyroscope acceleration}

Gyroscope acceleration value is calculated with the orientation force of the person. The speed of the movement is analyzed and the scale range differs for the walking position shown in the Figure 12.

Gyroscope $_{g}=\frac{\text { Gyroscope Value }}{\text { Sensitivity }} \mathrm{deg} / \mathrm{s} \rightarrow(4)$

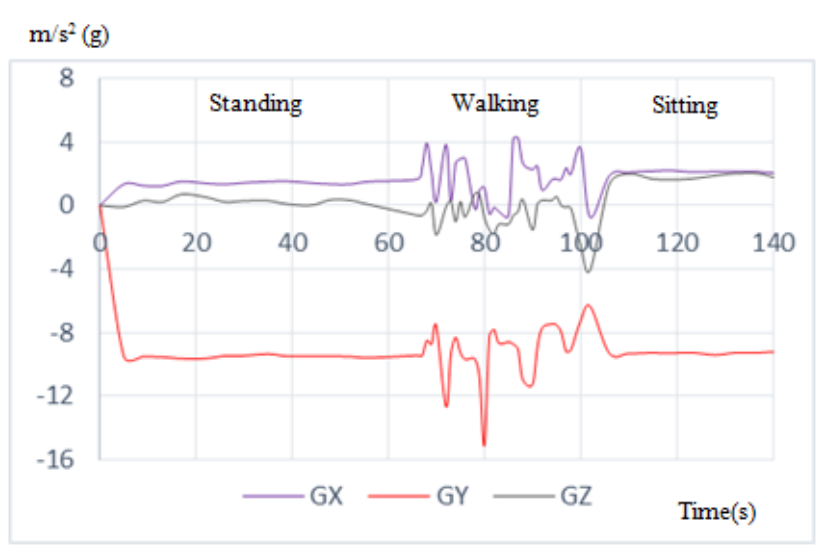

Figure 11. Gravity force in different stage.

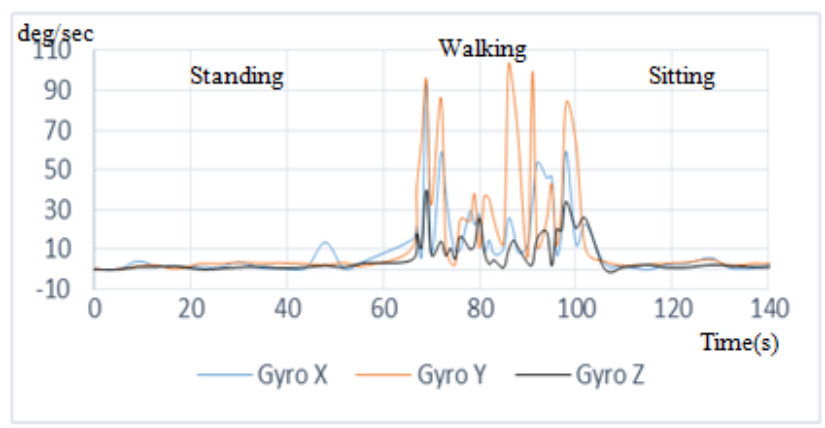

Figure 12. Gyroscope movement in different stage.

\section{Gait analysis}

Step detection is analyzed using the vector calculation of the raw sensor data with the average of previous ten data unit.

Vector $=\sqrt{\begin{array}{c}\left(A c c_{x}-A c c_{x a v g}\right)+\left(A c c_{y}-A c c_{y a v g}\right) \\ +\left(A c c_{z}-A c c_{z a v g}\right)\end{array}} \rightarrow(5)$

Once the vector is calculated, the average is taken between new vector and previous vector. If the average is greater than the threshold value in the MPU-6050, it counts the fall detection. From Table 3, the step detection experience with the accuracy percentage is shown.

Table 3. Step detection analysis.

\begin{tabular}{llll}
\hline $\begin{array}{l}\text { Gait } \\
\text { Analysis }\end{array}$ & $\begin{array}{l}\text { Actual } \\
\text { Steps }\end{array}$ & $\begin{array}{l}\text { Steps } \\
\text { Occurred }\end{array}$ & $\begin{array}{l}\text { Accuracy } \\
\text { Percentage }\end{array}$ \\
\hline Walking on slow speed & 50 & 46 & $92 \%$ \\
\hline Walking on faster speed & 50 & 44 & $88 \%$ \\
\hline Upstairs movement & 40 & 38 & $95 \%$ \\
\hline Downstairs movement & 40 & 36 & $90 \%$ \\
\hline
\end{tabular}

\section{Conclusion}

The design and implementation of the wearable sensing technology offers a novel solution for a comprehensive 
assessment to measure the associated degree of disability with low back pain. According to the user's comfort, the module can be placed near the lumbar spine or in the shirt pocket. The serial terminal Bluetooth application collects the data from the device and intimates the person when they are in a bad posture on angle below $70-75^{\circ}$ with accuracy of $95 \%$. The gait analysis with gravity acceleration and gyroscope acceleration unit provides an efficiency of $93 \%$. This initial prototype builds in a direction of development of an integrated, long-term monitoring device for the people working in a closed environment like software engineers.

\section{Acknowledgement}

The study was partially supported by Startup Mania Confederation of Indian Industry (CII), Yi Native lead, Native Angels Network and TBI@KEC under Erode Entrepreneurship Conclave 2017.

\section{References}

1. Chhikara A, Rice ASC, McGregor AH, Bello F. Wearable device for monitoring disability associated with low back pain. Reproduct Biol Anaesth (SORA) 2008; 16: 2817-2833.

2. El-Sayed B, Farra N, Moacdieh N, Hajj H, Haidar R. A novel mobile wireless sensing system for realtime monitoring of posture and spine stress. IEEE Trans Neural Syst Rehabil Eng 2011; 13: 18-23.

3. Brigante $\mathrm{CMN}$, Abbate $\mathrm{N}$, Basile A. Towards Miniaturization of a MEMS-Based Wearable Motion Capture System. IEEE Transact Industrial Electronics 2011; 58: 246-278.

4. Nemec D, Janota A, Hrubos M, Simak V. Intelligent RealTime MEMS Sensor Fusion and Calibration. IEEE Sensors J 2016; 16: 1558-1748.

5. Fachin F, Nikles S.A, Dugundji J.A, Wardle BL. Analytical extraction of residual stresses and gradients in MEMS structures with application to CMOS-layered materials. J Micromech Microeng 2011; 21: 095017.

6. Donatell GJ, Meister DW, O'Brien JR, Thurlow JS, Webster JG, Salvi FJ. A simple device to monitor flexion and lateral bending of the lumbar spine. IEEE Transact Neural Syst Rehab Eng 2005; 13: 1534-4320.

7. Garcia E, Ding H, Sarela A, Karunanithi M. Can a mobile phone be used as a pedometer in an outpatient cardiac rehabilitation program. IEEE/ICME Int Confer Complex Med Eng (CME) 2010.

8. Bertolotti GM, Cristiani AM, Colagiorgio P, Romano F, Bassani E, Caramia N, Ramat S. A wearable and modular inertial unit for measuring limb movements and balance control abilities. IEEE Sensors J 2016; 16: 1558-1748.

9. Liu H, Li R, Liu S, Tian S, Du J. SmartCare: Energyefficient long-term physical activity tracking using smartphones. Tsinghua Sci Technol 2015; 20: 348-363.

10. Alcaraz JC, Moghaddamnia S, Peissig J. An android-based application for digital gait performance analysis and rehabilitation. IEEE 17th International Conference on eHealth Networking, Applications and Services (Healthcom): Short and Demo Papers, 2015.

11. Ladenbauer J, Minassian K, Hofstoetter US, Dimitrijevic MR, Rattay F. Stimulation of the human lumbar spinal cord with implanted and surface electrodes: A computer stimulation study. IEEE Transact Neural Syst Rehab Eng 2010; 18: 1534-4320.

12. Tsai MH, Liu YC, Liang KC, Fang W. Monolithic CMOS -MEMS pure oxide tri-axis accelerometers for temperature stabilization and performance enhancement. J Microelectromech Syst 2015; 24: 1057-7157.

13. Millor N, Lecumberri P, Gomez M, Martınez-Ramırez A, Izquierdo $M$. Drift-free position estimation for periodic movements using inertial units. IEEE J Biomed Health Informat 2014; 18: 2168-2194.

14. Qu H, Fang D, Xie H. A single-crystal silicon 3-axis CMOS-MEMS accelerometer. Proc IEEE Sensors Conf, Vienna, Austria, 2004.

15. Samiei-Zonouz R, Memarzadeh-Tehran H, Rahmani R. Smartphone-centric human posture monitoring system. IEEE Canada Int Human Technol Confer (IHTC) 2014.

16. Shekeran SC, Sangeetha J. Analysis of age groups and motion speed using smart phone accelerometers. Int Confer Condition Assess Tech Elect Syst (CATCON) 2015.

17. Sun CM, Tsai MH, Liu YC, Fang W. Implementation of a monolithic single proof-mass tri-axis accelerometer using CMOS-MEMS technique. IEEE Trans Electron Devices 2010; 57: 1670-1679.

18. Xu W, Huang MC, Amini N, He L, Sarrafzadeh M. eCushion: A textile pressure sensor array design and calibration for sitting posture analysis. IEEE Sensors J 2013.

19. Bai YW, Wu SC, Tsai CL. Design and implementation of a fall monitor system by using a 3-axis accelerometer in a smart phone. IEEE 16th Int Symposium 2012.

20. Adachi Y, Miyamoto M, Kawai J. Improvement of SQUID magnetometer system for extending application of spinal cord evoked magnetic field measurement. IEEE Transact Appl Superconduct 2011; 21: 1051-8223.

21. Hu Y, Stoeling A, Wang YT, Zou Y, Sarrafzadeh M. Providing a cushion for wireless healthcare application development. IEEE Transact Digital Object Identifier 2010; 22: 278-664.

22. Zhong S, Wang L, Bernardos AM, Song M. An accurate and adaptive pedometer integrated in mobile health application. IET Int Confer Wireless Sensor Network 2010.

\section{*Correspondence to}

Maheswaran Shanmugam

Department of Electronics and Communication Engineering

Kongu Engineering College

India 